\title{
An IPv4-IPv6 Translation Mechanism for SIP Overlay Network in UMTS All-IP Environment
}

\author{
Whai-En Chen, Member, IEEE, Yi-Bing Lin, Fellow, IEEE, and Ai-Chun Pang, Member, IEEE
}

\begin{abstract}
Both IPv6 and session initiation protocol (SIP) are default protocols for Universal Mobile Telecommunications System (UMTS) all-Internet protocol (IP) network. In the existing mobile telecommunications environments, an IPv6-based UMTS all-IP network needs to interwork with other Internet protocol version 4 (IPv4)-based SIP networks. Therefore, mobile SIP applications are typically offered through an overlay structure over the IPv4-Internet protocol version 6 (IPv6) interworking environments. Based on 3GPP 23.228, we propose an IPv4-IPv6 translation mechanism (i.e., SIPv6 translator) that integrates different IP infrastructures (i.e., IPv4 and IPv6) to provide an overlay network for transparent SIP application deployment. In this paper, we present the architecture and the call flows of the SIPv6 translator. An analytic model is proposed to investigate the fault tolerance issue of our approach. Our study provides guidelines to select appropriate number of processors for fault tolerance.
\end{abstract}

Index Terms-All-IP, Internet protocol multimedia core network subsystem (IMS), Internet protocol version 4 (IPv4), Internet protocol version 6 (IPv6), session initiation protocol (SIP), Third-Generation Partnership Project (3GPP), translation.

\section{INTRODUCTION}

$\mathbf{T}$ HIRD-GENERATION Partnership Project (3GPP) defines Internet protocol multimedia core network subsystem (IMS) to support multimedia services [1]. To access various IMS services, Internet protocol version 6 (IPv6) is employed to provide large address space and new features such as mobility, security, quality-of-service (QoS), and plug-and-play. However, in the early stage of IPv6 deployment, many voice-over-IP (VoIP) networks still support Internet protocol version 4 (IPv4) only. Therefore, the IMS-application layer gateway (IMS-ALG) and the translation gateway ( $\mathrm{TrGW}$ ) are proposed to provide IPv4-IPv6 translation between the Universal Mobile Telecommunications System (UMTS) all-IP networks and the IPv4 session initiation protocol (SIP)-based VoIP networks. This structure supports IPv6 and IPv4 interworking infrastructures to provide an overlay network for transparent SIP application

\footnotetext{
Manuscript received December 1, 2004; revised June 20, 2005. This work was supported in part by the NICI IPv6 Project R0500 and P0500, in part by the National Science Council (NSC) Excellence Project under Grant NSC 93-2752-E-0090005-PAE, Grant NSC 93-2213-E-009-100, in part by the NTP VoIP Project under Grant NSC 92-2219-E-009-032, in part by IIS/Academia Sinica, and in part by ITRI/NCTU Joint Research Center.

W.-E. Chen and Y.-B. Lin are with the Department of Computer Science and Information Engineering, National Chiao Tung University, Hsinchu 300, Taiwan, R.O.C. (e-mail: wechen@csie.nctu.edu.tw; liny@ csie.nctu.edu.tw).

A.-C. Pang is with the Graduate Institute of Networking and Multimedia, National Taiwan University, Taipei 106, Taiwan, R.O.C. (e-mail: acpang@csie.ntu.edu.tw).

Digital Object Identifier 10.1109/JSAC.2005.856836
}

deployment. An interworking example of the UMTS all-IP network and the IPv4 VoIP network is given in Fig. 1.

In Fig. 1(a), radio access network (RAN) can be UMTS terrestrial radio access network (UTRAN) or Global System for Mobile Communication (GSM) enhanced data rates for global evolution (EDGE) radio access network (GERAN) [14]. General Packet Radio Service (GPRS) network [Fig. 1(b)] consists of serving GPRS support nodes (SGSNs) and gateway GPRS support nodes (GGSNs) that provide mobility and session management. The SGSN and the GGSN connect to RANs and external packet data networks (PDNs), respectively. The SGSN interworks with the UTRAN and the GEDGE through the $I u$ interface. An SGSN communicates with a GGSN through the $G n$ interface if both of them are in the same network and through the $G p$ interface if they are in different networks. A GGSN interacts with an external PDN through the $G i$ interface. $G n, G p$, and $G i$ are standard GPRS interfaces described in [12] and [15].

Home subscriber server (HSS) [see Fig. 1(c)] is the master database containing all UMTS user-related subscription information. An HSS consists of the IMS functionality and the home location register (HLR) functionality to support the Packet-switched (PS) and the circuit-switched (CS) domain services [2]. An SGSN communicates with the HSS through the $G r$ interface. A GGSN communicates with the HSS through the $G c$ interface. A mobile subscriber uses a mobile station (MS) or a user equipment (UE) to access the IMS services. To provide a data session for a UE, a connection between the UE and the GGSN is established. This connection is specified by a packet data protocol (PDP) context. The PDP context must be activated before a UE can access the IMS network.

A call session control function (CSCF) in IMS [Fig. 1(d)] is a SIP server responsible for call control. A CSCF can be a proxy-CSCF (P-CSCF), an interrogating-CSCF (I-CSCF), or a serving-CSCF (S-CSCF). The I-CSCF determines how to route incoming calls to the S-CSCF, and then to the destination UEs. That is, the I-CSCF serves as the contact point for the IMS network to hide the configuration, capacity, and topology of the IMS network from the outside world. When a UE attaches to the GPRS/IMS network and then performs PDP context activation, a P-CSCF is assigned to the UE. The P-CSCF contains limited address translation functions to forward the requests to the I-CSCF. Authorization for bearer resources in the network (where the UE visits) is performed by the P-CSCF. By exercising the IMS registration, an S-CSCF is assigned to serve the UE. This S-CSCF supports the signaling interactions with the UE for call setup and supplementary service control (e.g., service request and authentication). The S-CSCF also acts as a SIP registrar [9] to store the contact addresses of the UEs. 


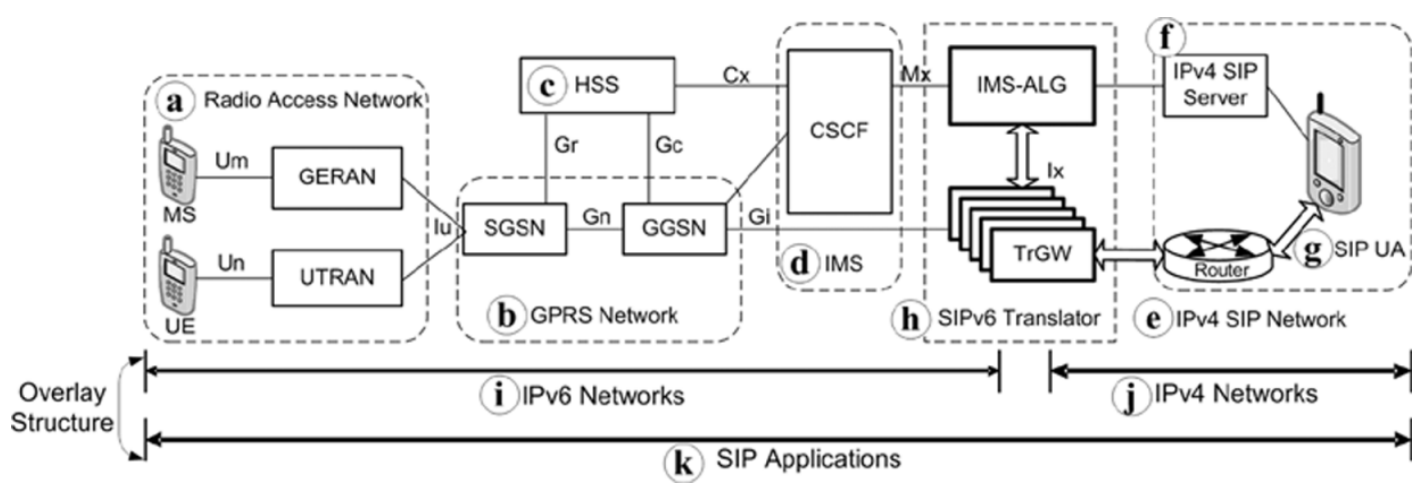

Fig. 1. Interworking between UMTS all-IP and the IPv4 VoIP networks.

The IPv4 SIP network [Fig. 1(e)] contains SIP servers [Fig. 1(f)] and SIP user agents (UAs) [Fig. 1(g)]. An SIP server forwards SIP request and response messages as a SIP proxy server, and also functions as a SIP registrar that stores the contact information of each SIP UA. The SIP server forwards SIP messages to a SIP UA according to the routing policy and the information stored in its registrar database. Via SIP, two UAs exchange the codec and connection information to set up a real-time transport protocol (RTP) [8] connection.

In a telecom-grade SIP VoIP network, each SIP UA (e.g., a UE or a SIP UA) is assigned a telephone number, and the SIP server (e.g., a CSCF or an IPv4 SIP server) is assigned a pool of telephone numbers. If a SIP UA's telephone number belongs to the telephone number pool of a SIP server, the UA should register to the registrar database of this SIP server. When the CSCF receives a SIP message with the Request-URI containing a telephone number in the IPv4 SIP server's pool, it forwards this SIP message to the IPv4 SIP server, and vice versa.

To provide data services to a UE, a connection between the UE and the GGSN should be established. This connection is specified by a PDP context. There are three types of PDP contexts including IPv4, IPv6, and point-to-point protocol (PPP). For a PDN directly connected to the GGSN, any of the above three protocols can be employed. Since 3GPP IMS is solely designed to use IPv6, a UE can only choose IPv6 as the type of the activated PDP context even if the UE is IPv4-IPv6 dual-stack capable. In other words, a SIP UA on 3GPP UE utilizes IPv6 exclusively to access the IMS.

Some non-UMTS all-IP networks [e.g., IPv4 SIP networks; see Fig. 1(j)] do not support IPv6 in the early stage of IPv6 deployment. By supporting IPv4-IPv6 interworking capability to provide an overlap network for transparent SIP application deployment, this paper proposes a SIPv6 translator [Fig. 1(h)] that consists of an IMS-ALG and a pool of TrGWs to translate SIP and RTP packets between IPv6 [i.e., IMS; see Fig. 1(i)] and IPv4 networks. The IMS-ALG communicates with TrGWs via the $I x$ interface and dispatches RTP streams to the appropriate TrGWs. In our implementation, the $I x$ interface is implemented by media gateway control protocol (MGCP) [6]. The IMS-ALG may re-assign the RTP connections to an available TrGW through SIP re-INVITE procedure if it detects failure of the original serving TrGW. To perform IPv4-IPv6 translation of SIP messages, the CSCF and the IPv4 SIP server set the IMS-ALG as their outbound proxy. With the outbound proxy setting, the CSCF always forwards a SIP message to the IMS-ALG before sending it to the external IPv4 SIP server, and vice versa. In this paper, we present the architecture and call flows of the SIPv6 translator, and then analyze the availability of the SIPv6 translator through an analytic model.

\section{SIPV6 TRANSLATOR}

Since the volume of RTP traffic is much larger than that of SIP signaling traffic, we propose a SIPv6 translator architecture that can effectively handle the RTP traffic, as well as the SIP signaling. Details of the SIPv6 translator in Fig. 1(h) are illustrated in Fig. 2. The SIPv6 translator architecture consists of an IMS-ALG and a pool of TrGWs. The IMS-ALG translates IPv4-IPv6 formats of the SIP messages including the session description protocol (SDP) [10] fields. On the other hand, a TrGW translates the formats of the RTP packets. Our approach utilizes a pool of TrGWs to increase the transmission bandwidth for RTP traffic. The TrGW client [Fig. 2(e)] at the IMS-ALG communicates with the TrGW daemon [Fig. 2(i)] at the TrGW through the $I x$ Interface.

The IMS-ALG consists of an IPv4 module [Fig. 2(a)], an IPv6 module [Fig. 2(b)], a back-to-back UA (B2BUA) [Fig. 2(c)], a URL mapping table [Fig. 2(d)], and a TrGW client [Fig. 2(e)]. The IPv4 and the IPv6 modules bind on IPv4 and IPv6 sockets, respectively. They are responsible for transmitting/receiving packets to/from the IPv4 and IPv6 networks. By interacting with the IPv4 and the IPv6 modules, the B2BUA contains a user agent server (UAS) and a user agent client (UAC). The B2BUA breaks the SIP session between the IPv6 and IPv4 SIP UAs into an IPv6 SIP subsession and an IPv4 SIP subsession. The URL mapping table stores IPv4-IPv6 call leg (i.e., SIP header fields Call-ID, From, To, and Via) mappings for the IPv4 and IPv6 SIP subsessions. The IMS-ALG instructs the TrGW to obtain the IP/port mappings through the TrGW client. To translate the RTP packets, the TrGW implements a network address (port multiplexing) translation and protocol translation [NA(P)T-PT [7], [13]] that consists of a forwarding engine [Fig. 2(f)], a stateless IP/Internet control message protocol (ICMP) translation (SIIT) component [5] [Fig. 2(g)], an address mapper [Fig. 2(h)], and a TrGW daemon [Fig. 2(i)]. The forwarding engine dispatches the incoming RTP packets 


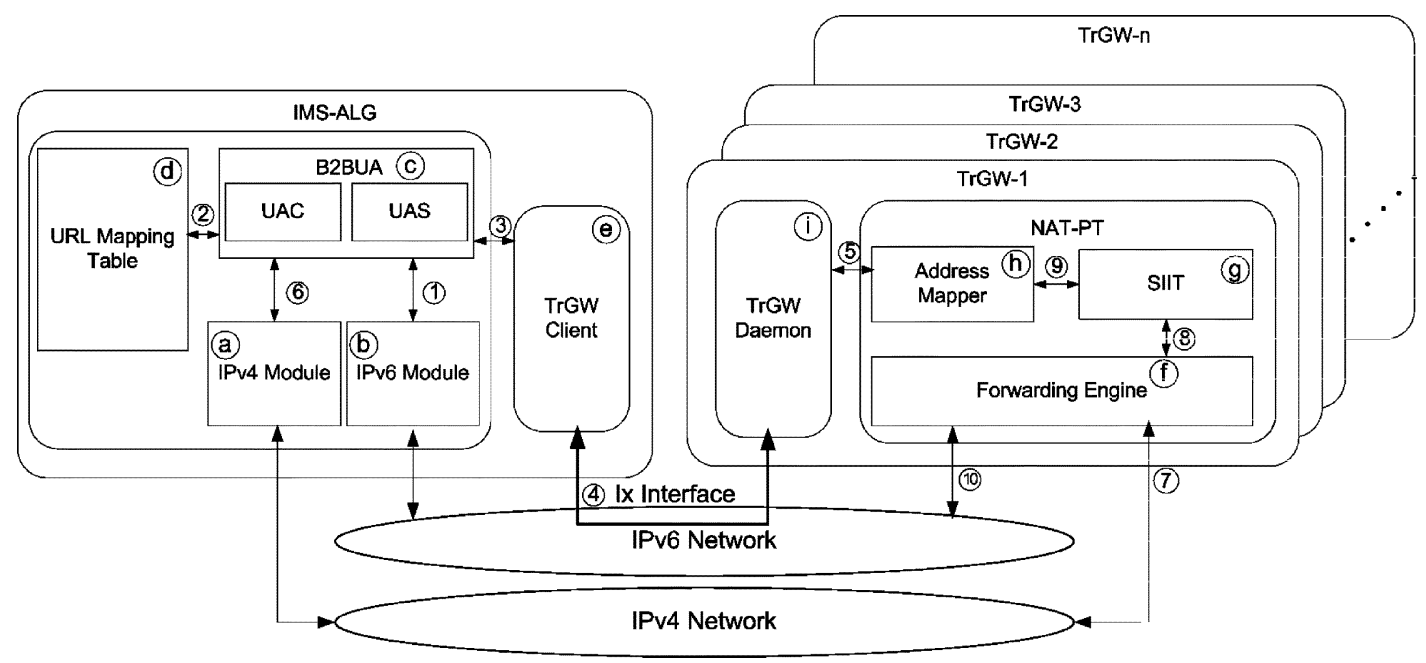

Fig. 2. SIPv6 translator architecture.

to the SIIT component for packet format translation and then sends the translated RTP packets to the IPv4/IPv6 network. The SIIT component translates the IP and ICMP headers according to IETF Specification RFC2765 [5]. The address mapper contains an IPv4-IPv6 mapping table and a pool of public IPv4 addresses (e.g., 140.113.1.10 140.113.1.20) that are assigned to the TrGW.

The IPv4-IPv6 format translation procedures are described as follows. When the B2BUA receives a SIP INVITE message from the IPv6 module [Fig. 2(1)], it acts as a UAS and retrieves the SIP header fields (i.e., Call-ID, From, To, and Via) of this message. The B2BUA stores these header fields into the URL mapping table [Fig. 2(2)]. Then, it creates a new IPv4 SIP message and an IPv4 call leg by using the IPv4 address and port number of the IPv4 module [i.e., 140.113.1.1:5060; see Fig. 2(a)]. The B2BUA stores the new call leg in the URL mapping table. The INVITE message contains the SDP fields that include IPv6 address and port number information for RTP packets. Therefore, IPv6 to IPv4 translation is required for the SDP fields. The B2BUA invokes a selected TrGW to obtain the mapped IPv4 address and port number for the SDP $c$ and $m$ fields [Fig. 2(3) and (4)]. The TrGW daemon invokes the address mapper [Fig. 2(5)] to build an IP/port mapping and replies the mapped IP address and port number to the IMS-ALG. This mapping will be used for RTP packet translation later. Finally, the B2BUA modifies the SDP fields and acts as a UAC to set up a call path to the called UA through the IPv4 module [Fig. 2(6)]. After the call session is set up by SIP signaling, the RTP packets are delivered between the UE and the IPv4 SIP UA through the selected TrGW. Upon receipt of an IPv4 RTP packet [Fig. 2(7)], the forwarding engine of the $\mathrm{TrGW}$ dispatches the incoming RTP packet to the SIIT component to translate the IP address and port number of the RTP packet [Fig. 2(8)]. The SIIT component retrieves the mapped IPv6 address and port number from the address mapper [Fig. 2(9)], translates the format of RTP packet, and returns it to the forwarding engine. Then, the forwarding engine sends the translated packet to the IPv6 network [Fig. 2(10)]. The detailed call setup and termination signaling flows are described in the following sections.

\section{A. IPv6-to-IPv4 Call Setup Flow}

Fig. 3 presents the call setup flow between an IMS calling party (UE) and an IPv4 VoIP called party (UA). In this scenario, the UE is assigned an IPv6 address 3ffe:3600:1::3, port number 5060 for SIP and port number 9000 for RTP. The UA is assigned an IPv4 address 140.113.1.2, port number 5060 for SIP and port number 8002 for RTP. The telephone numbers ranging from 0944000000 to 0944000999 are assigned to the SIPv4 proxy and the UA is assigned the telephone number 0944000000 . Initially, the UA registers its IP address to the SIPv4 proxy and sets the SIPv4 proxy as its outbound proxy. Similarly, the telephone numbers ranging from 0944001000 to 0944001999 are assigned to the CSCF. The UE is assigned the telephone number 0944001111 and therefore sets the CSCF as its outbound proxy. The address mapper of the TrGW contains a pool of public IPv4 addresses ranging from 140.113.1.10 to 140.113.1.20, and a global IPv6 address 3ffe:3600:2::1. Table I shows the IPv4 address, the IPv6 address and the telephone number of these components illustrated in Fig. 3. For the demonstration purposes, we use the component names to denote the IPv6 and IPv4 addresses of the components. For example, "SIPv4_Proxy(v6)" and "SIPv4_proxy(v4)" denote the IPv6 and IPv4 addresses (i.e., 3ffe:3600:1::140.113.131.5 and 140.113.1.5) of SIPv4 server, respectively.

Step 1) To set up a call to the UA, the UE first sends an INVITE message to the CSCF, where the Request-URI is $0944000000 @ \mathrm{CSCF}$, the Via header field is SIP/2.0/UDP UE:5060, the IPv6 address in the SDP $c$ field is UE (i.e., $3 f f e: 3600: 1:: 3$ ), and the port number in the SDP $m$ field is 9000 .

Step 2) When the CSCF receives the INVITE message, it checks the telephone number in the $R e$ quest-URI. Since the number 0944000000 belongs to the telephone number pool of the SIPv4 proxy, the CSCF modifies the Request-URI to 0944000000@SIPv4_Proxy(v6), inserts the Via header field with the CSCF address (i.e., 


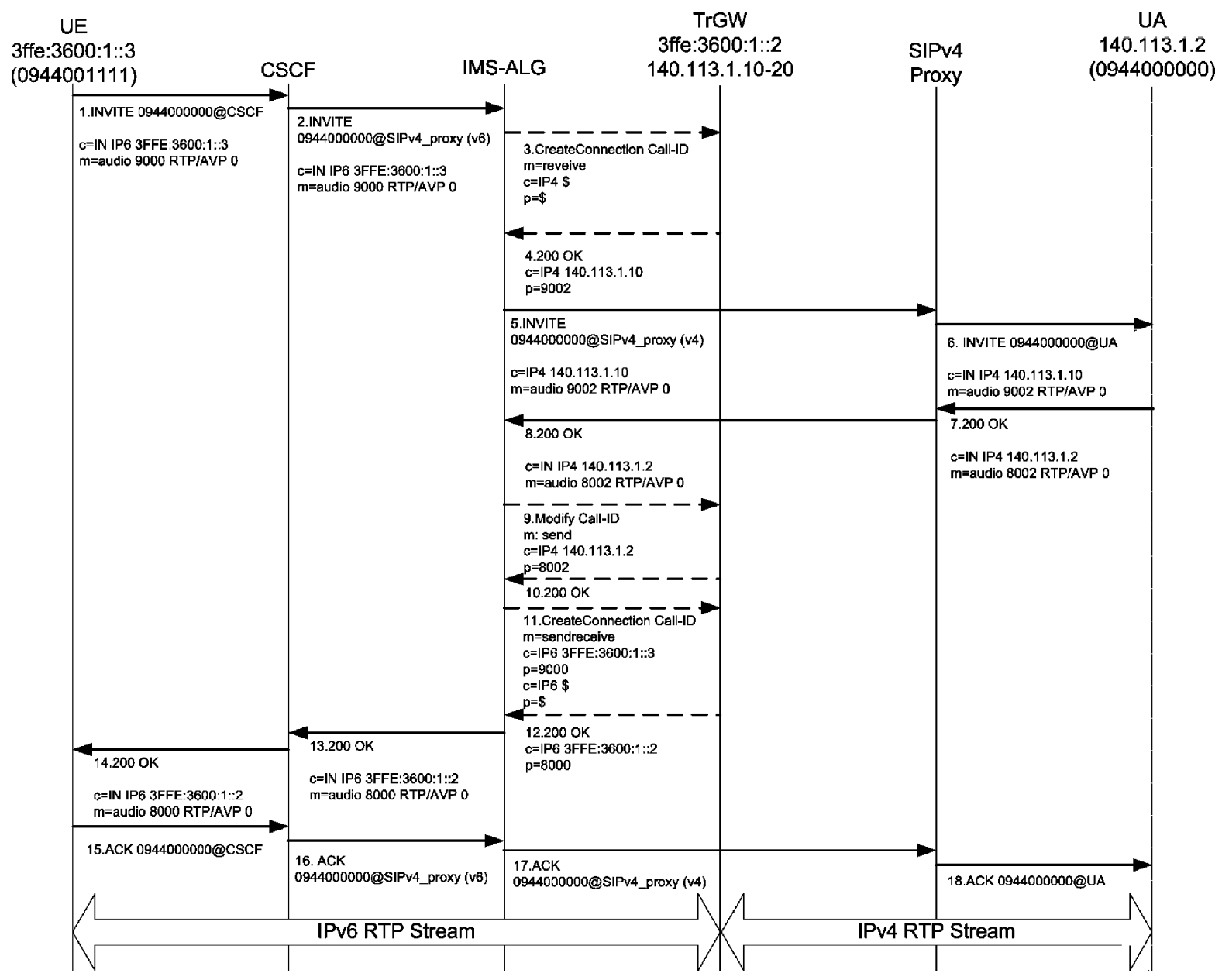

Fig. 3. Call setup flow.

SIP/2.0/UDP CSCF:5060), and then forwards this message to its outbound proxy; i.e., the IMS-ALG.

Step 3) When the IMS-ALG receives the INVITE message, it instructs a TrGW to generate the IPv4 address and port number in the SDP $c$ and $m$ fields for the subsequent RTP packet transmission. The IMS-ALG selects an available TrGW and instructs the $\mathrm{TrGW}$ to build an IP/port mapping in the address mapper through MGCP CreateConnection message [Fig. 2(3), (4), and (5)].

Step 4) The TrGW returns the mapped IPv4 address (140.113.1.10 representing the address of the TrGW) and port number (9002) in an MGCP $200 \mathrm{OK}^{1}$ message. When the IMS-ALG receives the SIP 200 OK message, it retrieves the IP address and port number from this message. This IPv4 information will be filled in the SDP $c$ and $m$ fields of the IPv4 INVITE message to be created in the next step.

Step 5) The IMS-ALG translates the Request-URI [0944000000@SIPv4_proxy(v6)] to 0944

${ }^{1}$ Note that both MGCP and SIP have defined 200 OK messages. The MGCP 200 OK is sent from the TrGW to the IMS-ALG only.
TABLE I

IPv4 ADDRESS, IPv6 ADDRESS, AND TELEPHONE NUMBER

\begin{tabular}{|c|c|c|c|}
\hline Device & $\begin{array}{l}\text { IPv4 } \\
\text { Address }\end{array}$ & IPv6 Address & $\begin{array}{l}\text { Telephone } \\
\text { Number }\end{array}$ \\
\hline CSCF & - & 3ffe: $3600: 1:: 1$ & $\begin{array}{l}0944001000 \text { to } \\
0944001999\end{array}$ \\
\hline IMS-ALG & 140.113.1.6 & $3 \mathrm{ffe}: 3600: 1:: 6$ & - \\
\hline $\begin{array}{l}\text { SIPv4 } \\
\text { Proxy }\end{array}$ & 140.113.1.5 & 3 ffe $: 3600: 1:: 140.113 .131 .5$ & $\begin{array}{l}0944000000 \text { to } \\
0944000999\end{array}$ \\
\hline TrGW & $\begin{array}{l}140.113 .1 .10 \\
\text { to } \\
140.113 .1 .20\end{array}$ & 3ffe:3600:1::2 & - \\
\hline UA & 140.113 .1 .2 & - & 0944000000 \\
\hline UE & - & 3ffe: $3600: 1:: 3$ & 0944001111 \\
\hline
\end{tabular}

000000@SIPv4_proxy(v4) and generates a new IPv4 INVITE message by using its IPv4 address. Therefore, the Via header field of this IPv4 INVITE message is SIP/2.0/UDP IMS-ALG:5060. The IMS-ALG fills the IP address (140.113.1.10) and port number (9002) obtained from the $\operatorname{TrGW}$ into the SDP $c$ and $m$ fields. Then, the message is sent to the SIPv4 proxy according to the Request-URI.

Step 6) Upon receipt of the INVITE message, the SIPv4 proxy retrieves the current IPv4 address of the destination UA from its 


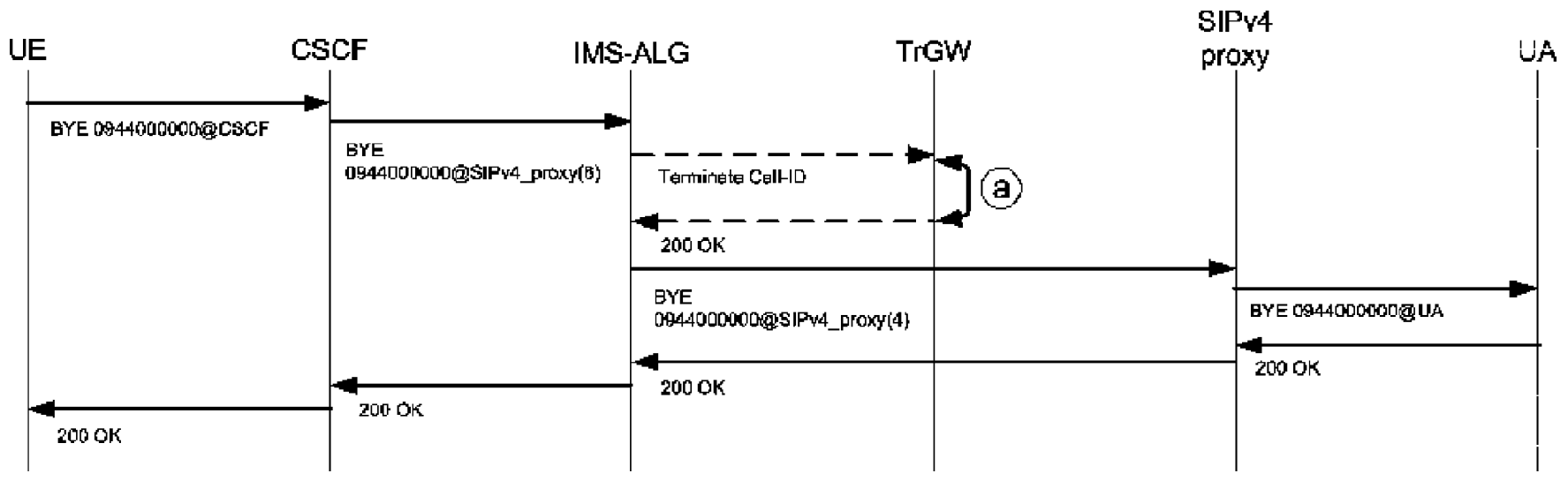

Fig. 4. Call termination flow.

Step 7) When the UA receives the INVITE message, it obtains the RTP information of the UE from the SDP $c$ and $m$ fields. After the called party has picked up the phone, the UA sends a SIP 200 OK message to the UE. This message carries the IP address (140.113.1.2) and port number (8002) of the RTP connection in the SDP $c$ and $m$ fields. The $200 \mathrm{OK}$ message is sent back to the SIPv4 proxy according to the top Via header field (SIP/2.0/UDP SIPv4_proxy(4):5060). Therefore, the route of the 200 OK message is the reverse of that for the INVITE message.

Step 8) When the SIPv4 proxy receives the 200 OK message, it removes the top Via header field and forwards the 200 OK message to the IMS-ALG according to the next Via header field (SIP/2.0/UDP IMS-ALG:5060).

Step 9) Upon receipt of the SIP 200 OK message, the IMS-ALG obtains the RTP information of the UA from the SDP $c$ and $m$ fields (i.e., the IP address is UA or 140.113.1.2 and port number is 8002) and sends this information to the TrGW through MGCP Modify message. [Fig. 2(3), (4), and (5)].

Step 10) The TrGW replies an MGCP 200 OK message after it has built the IPv4/port mapping (i.e., the UA's address UA:8002 is mapped to an address of the TrGW, 140.113.1.10:9002). At this point, the IPv4/port mapping is established for the IPv4 RTP packet transmission through the TrGW.

Step 11) The IMS-ALG instructs the TrGW to send and receive the IPv6 RTP packets. The IMS-ALG sends the UE's IPv6 address (3ffe:3600:1::3) and port number (9000) to the $\mathrm{TrGW}$ and invokes the TrGW to build an IPv6/port mapping.
Step 12) The TrGW returns the mapped TrGW's IPv6 address (3ffe:3600:1::2) and port number (8000) to the IMS-ALG through the MGCP 200 OK message. Then, the IMS-ALG modifies the SDP $c$ and $m$ fields of the SIP $200 \mathrm{OK}$ message.

Step 13) The SIP 200 OK message is sent back to the CSCF according to the top Via header field (SIP/2.0/UDP CSCF:5060) retrieved from URL mapping table of the IMS-ALG.

Step 14) Upon receipt of the 200 OK message, the CSCF removes the top Via header field (SIP/2.0/UDP CSCF:5060) and forwards the message to the UE according to the next Via header field (SIP/2.0/UDP UE:5060).

Step 15-18) The ACK message is returned to the UA. The IPv4-IPv6 translation of the ACK message is similar to that of the INVITE message, and the details are omitted.

After Step 18), the RTP connection is established between the UE and the UA through the TrGW assigned by the IMS-ALG.

\section{B. IPv6-to-IPv4 Call Termination Flow}

Fig. 4 shows the call termination flow initiated by the UE. In this example, the UE sends an IPv6 BYE message to terminate the call. When the IMS-ALG receives the BYE message, it invokes the TrGW to terminate the RTP connection based on the Call-ID [Fig. 4(a)] created at Step 3) in Fig. 3. The TrGW replies an MGCP 200 OK message after the RTP connection is successfully terminated. Then, the IMS-ALG forwards an IPv4 BYE message to the UA through the SIPv4 proxy. After the UA has received the IPv4 BYE message, it terminates the call, and replies an IPv4 SIP 200 OK message to the UE. Finally, the UE receives the translated SIP 200 OK message and the call is terminated. The IPv4-to-IPv6 call setup and termination procedures are similar to the IPv6-to-IPv4 procedures and the details are omitted.

\section{Fault Tolerance of The SIPv6 TRANSLAToR}

This section investigates fault tolerance of the SIPv6 Translator. Basically, availability of our approach can be achieved 


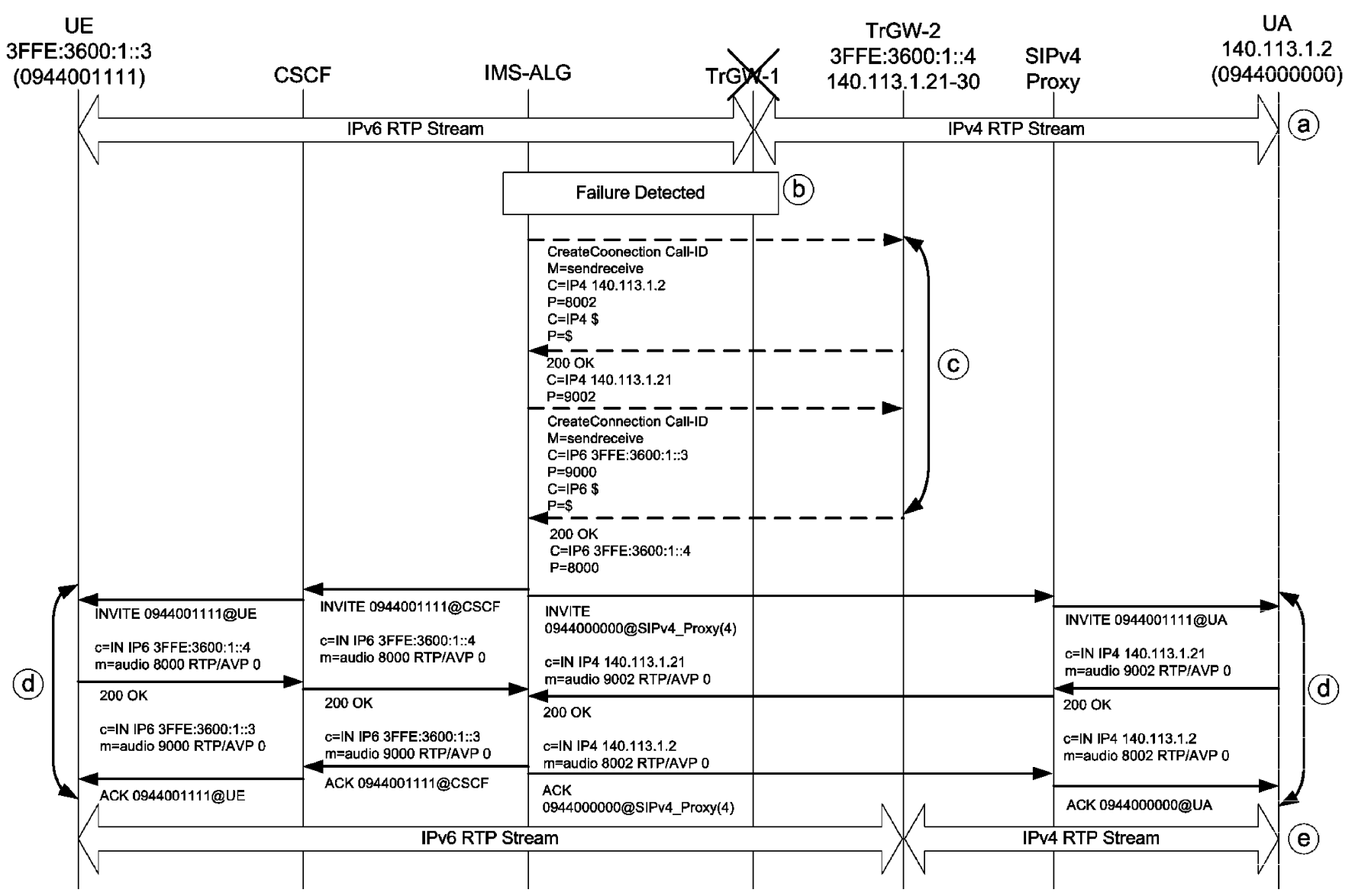

Fig. 5. Message flow for RTP session migration.

through two configurations: Configuration I consists of multiple TrGWs and Configuration II includes a single TrGW with multiple processors. We can also combine both Configurations I and II to come out a hybrid configuration.

\section{A. RTP Migration for Configuration I}

Consider a call session between a UA and a UE indirectly through a TrGW called TrGW-1. After call setup, the established RTP session is divided into an IPv6 RTP subsession between the UE and TrGW-1 and an IPv4 RTP subsession between TrGW-1 and the UA [see Fig. 5(a)]. Since the IMS-ALG processes all SIP signaling messages (including the SDP fields), it maintains the information of all RTP connections. If TrGW-1 fails, the IMS-ALG will detect the situation through the $I x$ interface [Fig. 5(b)], and starts to migrate the RTP sessions from TrGW-1 to other available TrGWs; e.g., TrGW-2 in Fig. 5. Specifically, the IMS-ALG instructs TrGW-2 (through MGCP CreateConnection and 200 OK messages) to create the IPv4 and IPv6 addresses and ports mappings in its address mapper [Fig. 5(c)]. After the IMS-ALG obtains the IP/port mappings, it informs the UE and the UA to migrate the RTP subsessions from TrGW-1 to TrGW-2 through SDP exchange [Fig. 5(d)]. The IMS-ALG then sends the INVITE messages with the IPv6 and IPv4 mappings to the UE and the UA, respectively. Upon receipt of the INVITE messages, the UE and the UA obtain the IP address and port information of TrGW-2 from the SDP portion and then migrate the RTP subsessions to TrGW-2. This

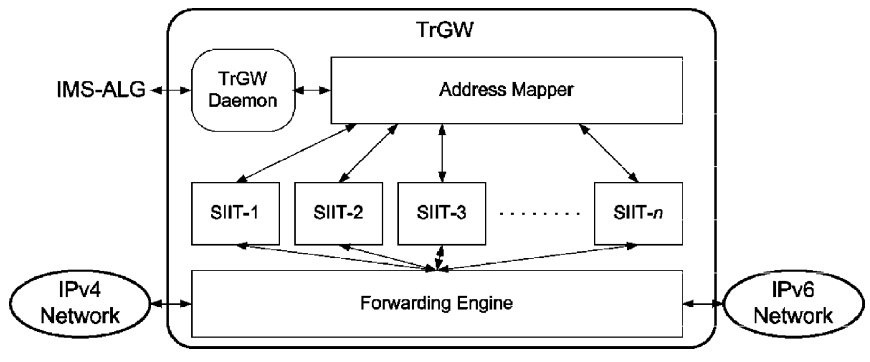

Fig. 6. Architecture of single TrGW with multiple processors.

procedure is called re-INVITE. After re-INVITE, the RTP session is migrated to TrGW-2 [Fig. 5(e)].

\section{B. Configuration II: Single TrGW With Multiple Processors}

This configuration is a special case of the SIPv6 Translator (Fig. 6) with only one TrGW. In this configuration, there is a forwarding engine, a TrGW daemon, an address mapper, and $n$ SIIT components. For SIP message format translation, the $\mathrm{TrGW}$ daemon and the address mapper are involved as described before. For an incoming RTP packet, the forwarding engine sends the packet to an idle SIIT component for processing. Therefore, the TrGW daemon and the forwarding engine may be executed by a fast processor, and each SIIT component is run on an individual processor. The address mapper can be simultaneously accessed by multiple SIIT components (there is no multiple read/write issues). If a SIIT 
component fails, the forwarding engine will forward packets to other available SIIT components.

\section{FAUlT TOLERANCE OF THE SIPV6 TRANSLATOR}

This section proposes analytic models to investigate Configurations I and II for the TrGWs. We first describe the analytic model for Configuration II based on the techniques we developed in [4], and then extend the model for Configuration I.

In Configuration II, we assume that there are $n$ processors in the TrGW. A processor is either operational or down. The operational readiness of a processor is modeled by alternating renewal processes. In the $k$ th renewal cycle $(k \geq 1)$, let $X_{k}$ be the operational time and $Y_{k}$ be the downtime. Assume that the random vectors $\left(X_{k}, Y_{k}\right)$ are independent and identically distributed. Note that $Y_{k}$ may be dependent on $X_{k}$ (i.e., the length of the down time may depend on the previous operating time). If $\mathrm{E}\left[X_{k}+Y_{k}\right]<\infty$ and $X_{k}+Y_{k}$ is nonlattice, ${ }^{2}$ then the operational readiness (see [11, Th. 3.4.4]) for a processor is

$p=\lim _{t \rightarrow \infty} \operatorname{Pr}[$ A processor is operational at time $t]$

$$
=\frac{E\left[X_{k}\right]}{E\left[X_{k}\right]+E\left[Y_{k}\right]}
$$

If the TrGW is an $n$-processor system, then the

probability that $i$ processors are operational is $P(n, i)$

$$
=\left(\begin{array}{c}
n \\
i
\end{array}\right) p^{i}(1-p)^{n-i}
$$

where $i \leq n$. We are interested in the following two measures of the TrGW.

- When a RTP packet arrives, what is the probability $\alpha_{n}$ that a TrGW with $n$ processors is down (i.e., $i=0$ in Configuration II)?

- If the TrGW is operational (i.e., $i>0$ ), what is the expected response time $\overline{R_{I I}}=\mathrm{E}\left[R_{I I} \mid i>0\right]$ to process a RTP packet at the TrGW (where $R_{I I}$ is the response time of a RTP packet in Configuration II)?

We assume that the RTP packet arrivals to the SIPv6 translator form a Poisson connection with rate $\lambda$. The service time $t_{s}$ (i.e., the single RTP packet processing time at a processor) has a general distribution with mean $1 / \mu$. Therefore, the TrGW can be modeled by an $\mathrm{M} / \mathrm{G} / i$ queue, where $i$ is the number of operational processors. Although no closed form expression for the mean query response time $\mathrm{E}\left[\mathrm{R}_{I I, i}\right]$ is known, it can be approximated as [12]

$$
\mathrm{E}\left[\mathrm{R}_{I I, i}\right] \cong \frac{\left(1+c_{v}^{2}\right) \rho_{i} \theta_{i}}{2 \lambda\left(1-\rho_{i}\right)}+\frac{1}{\mu}
$$

where $\rho_{i}=\lambda / i \mu$

$$
\begin{aligned}
\theta_{i}=\frac{\lambda^{i}}{\mu^{i-1}(i-1) !(i \mu-\lambda)} & \\
& \times\left[\sum_{j=0}^{i-1} \frac{\lambda^{j}}{\mu^{j} j !}+\frac{\lambda^{i}}{\mu^{i-1}(i-1) !(i \mu-\lambda)}\right]^{-1}
\end{aligned}
$$

\footnotetext{
${ }^{2} \mathrm{~A}$ nonnegative random variable is said to be lattice if it only takes on integral multiples of some nonnegative number.
}

and $c_{v}$ is the coefficient of variation of the RTP packet processing time distribution. Note that $c_{v}=\mu \sqrt{\operatorname{Var}\left[t_{s}\right]}$, where $\operatorname{Var}\left[t_{s}\right]$ is the variance of $t_{s}$. Note that (2) is exact for $\mathrm{M} / \mathrm{M} / i$ and $\mathrm{M} / \mathrm{G} / 1$.

From (1), it is clear that

$$
\alpha_{n}=P(n, 0)=(1-p)^{n} .
$$

From (2)

$$
\begin{aligned}
\overline{R_{I I}} & =\left[\frac{1}{\sum_{i=1}^{n} P(n, i)}\right]\left\{\sum_{i=1}^{n} E\left[R_{I I, i}\right] P(n, i)\right\} \\
& \cong\left(\frac{1}{1-\alpha_{n}}\right)\left\{\sum_{i=1}^{n}\left[\frac{\left(1+c_{v}^{2}\right) \rho_{i} \theta_{i}}{2 \lambda\left(1-\rho_{i}\right)}+\frac{1}{\mu}\right]\left(\begin{array}{l}
n \\
i
\end{array}\right) p^{i}(1-p)^{n-i}\right\} .
\end{aligned}
$$

For Configuration I, the output measures can be expressed as the same way as those for Configuration II except that the derivation for $\mathrm{E}\left[\mathrm{R}_{I, i}\right]$ is different from $\mathrm{E}\left[\mathrm{R}_{I I, i}\right]$. In Configuration I, every TrGW operates independently. Suppose that the TrGW migration procedure described in the previous section exercises load balancing strategy; that is, when a TrGW fails, the workload of this failed TrGW is evenly distributed to the remaining operational TrGWs. In this case, the RTP packet arrival rate to a TrGW is $\lambda_{i}=\lambda / i$, if there are $i$ operational TrGWs. Therefore, for the $n$-TrGW SIPv6 translator with $i$ operational TrGW (where $i \leq n$ ), each TrGW can be modeled by an M/G/1 queue with RTP packet arrival rate $\lambda_{i}$ and the service time $t_{s}$ has a general distribution with mean $1 / \mu$. Therefore, $\mathrm{E}\left[\mathrm{R}_{I, i}\right]$ for Configuration I can be expressed as

$$
E\left[R_{I, i}\right]=\frac{\rho_{i}\left(1+c_{v}^{2}\right)}{2 \mu\left(1-\rho_{i}\right)}+\frac{1}{\mu}, \text { where } \rho_{i}=\frac{\lambda_{i}}{\mu}=\frac{\lambda}{i \mu} .
$$

From (5) and (3)

$$
\begin{aligned}
\overline{R_{I}} & =\left[\frac{1}{\sum_{i=1}^{n} p(n, i)}\right]\left\{\sum_{i=1}^{n} E\left[R_{I, i}\right] P(n, i)\right\} \\
& =\left(\frac{1}{1-\alpha_{n}}\right)\left\{\sum_{i=1}^{n}\left[\frac{\rho_{i}\left(1+c_{v}^{2}\right)}{2 \mu\left(1-\rho_{i}\right)}+\frac{1}{\mu}\right]\left(\begin{array}{c}
n \\
i
\end{array}\right) p^{i}(1-p)^{n-i}\right\} .
\end{aligned}
$$

\section{NUMERICAL RESULTS}

Based on the analytic model developed in the previous section, we use numerical examples to illustrate the effects of $n$ (the number of TrGWs in Configuration I or the number of processors in Configuration II), $p$ (the steady-state probability that a TrGW/processor is operational), $\lambda$ (the net RTP packet arrival rate), and $c_{v}$ (the coefficient of variation of the service time $t_{s}$ ) on the output measures $\overline{R_{I}}$ and $\overline{R_{I I}}$ [the expected RTP packet response times for Configurations I and II; see (4) and (6)].

Fig. 7 plots $\overline{R_{I}}$ and $\overline{R_{I I}}$ as functions of $n, p$ and $\lambda$, where $c_{v}=1.0$ (i.e., the RTP packet processing time $t_{s}$ is exponentially distributed), and $p=0.8,0.9$ and 0.99999 . Note that $p=0.99999$ represents that the TrGW is a highly reliable telecom-grade equipment and $p=0.8$ may represent that the 


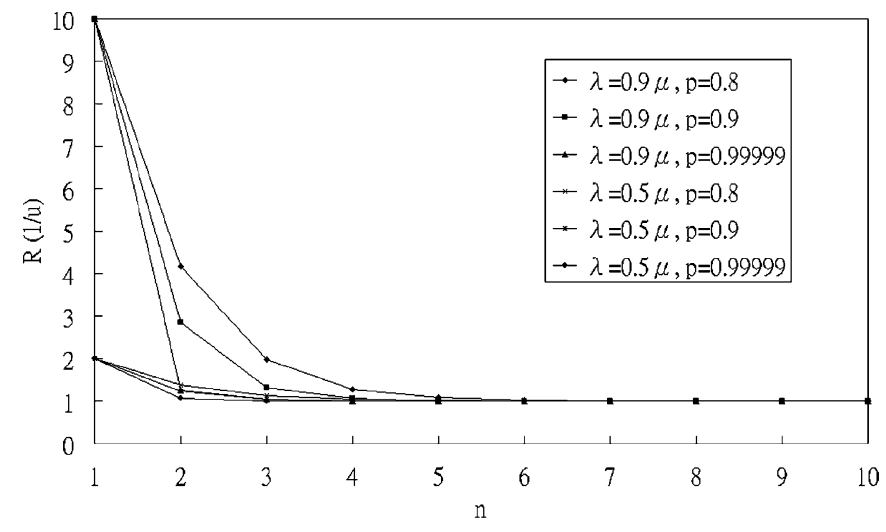

(a)

Fig. 7. Effects of $n, p$, and $\lambda$. (a) Configuration I. (b) Configuration II.

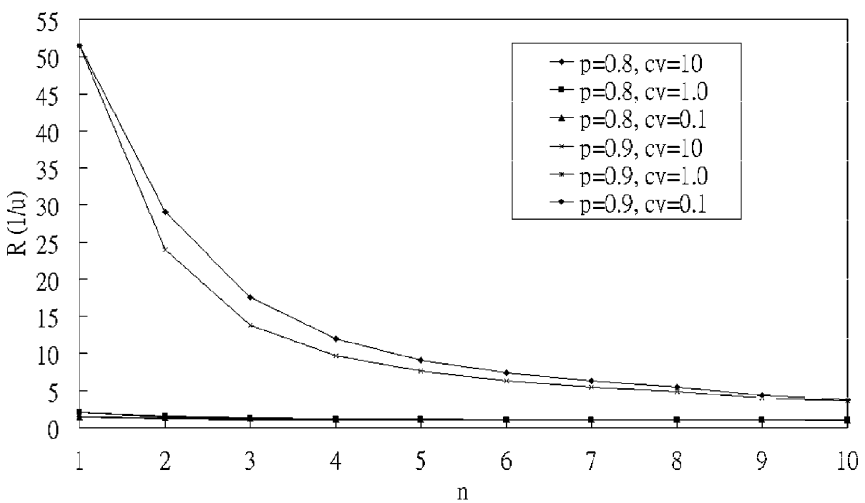

(a)

Fig. 8. Effects of $c_{v}(\lambda=0.5 \mu)$. (a) Configuration I. (b) Configuration II.

TrGW is a normal Internet network equipment. Fig. 7 shows intuitive results that $\overline{R_{I}}$ and $\overline{R_{I I}}$ are decreasing functions of $n$, and are increasing functions of $\lambda$. We also observe the following nontrivial results: $\overline{R_{I}}$ and $\overline{R_{I I}}$ are significantly affected by the change of $n$ when $n$ is small (e.g., $n \leq 4$ for the case when $\lambda=0.9 \mu$ ). On the other hand, when $n$ is large, $\overline{R_{I}}$ and $\overline{R_{I I}}$ are only insignificantly affected by the change of $n$, and the $\overline{R_{I}}$ $\left(\overline{R_{I I}}\right)$ value approximates $1 / \mu$. Specifically, for $n \geq 5$, adding extra TrGWs will not improve the performance of the SIPv6 translator. Fig. 7(a) and (b) also indicates that increasing $p$ results in the increase of $i$ (i.e., the number of operational TrGWs or processors) and, thus, the decrease of the expected response time $\overline{R_{I}}\left(\overline{R_{I I}}\right)$. Note that when $n=1, \overline{R_{I}}$ and $\overline{R_{I I}}$ are not affected by the $p$ value because we assume that one TrGW or processor is always operational.

Fig. 8 plots $\overline{R_{I}}$ and $\overline{R_{I I}}$ against $c_{v}$ (i.e., the coefficient of variation of the RTP packet processing time distribution). In Fig. 8, $\lambda=0.5 \mu$, and $p=0.8$ and 0.9 . The curves indicate that $\overline{R_{I}}$ $\left(\overline{R_{I I}}\right)$ increases as $c_{v}$ increases. This phenomenon is explained as follows. As $c_{v}$ increases (i.e., variance of $t_{s}$ increases), more long and short RTP packet processing times are observed. A RTP packet requiring long processing time at the TrGW results in longer queueing delay of subsequent packet processing even if these subsequent packets require short processing times. Thus, larger $\overline{R_{I}}$ and $\overline{R_{I I}}$ are observed. Also when $c_{v} \leq 1, c_{v}$ only has insignificant effect on both $\overline{R_{I}}$ and $\overline{R_{I I}}$. On the other hand, when $c_{v} \geq 1, \overline{R_{I}}$ and $\overline{R_{I I}}$ significantly increase as $c_{v}$

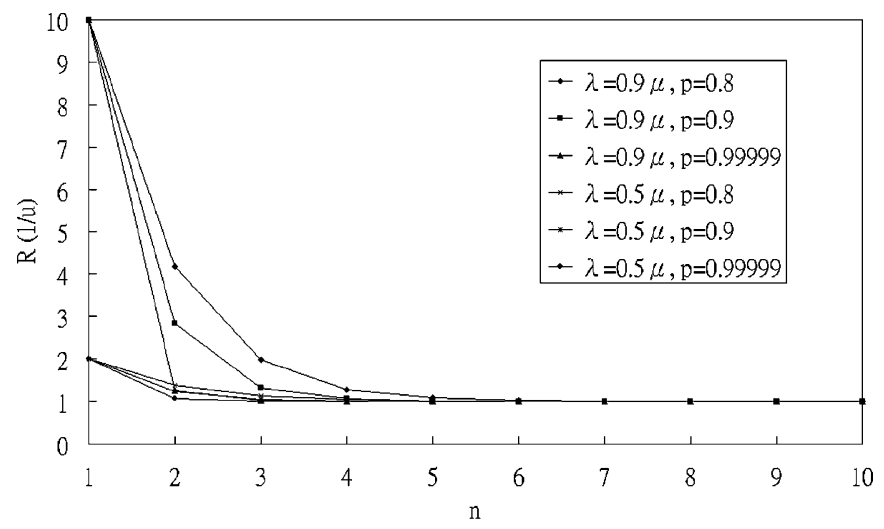

(b)

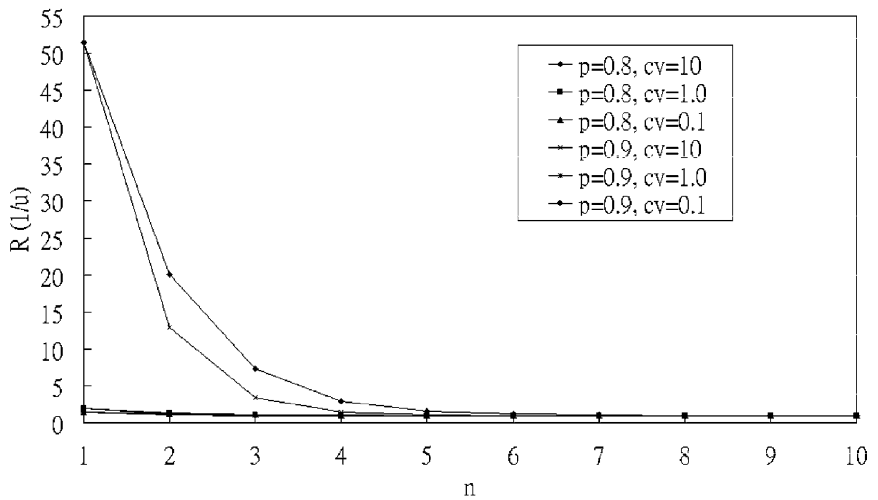

(b)

TABLE II

EXPECTED RESPONSE TIME AND THE NUMBER OF REQUIRED TRGWS FOR DIFFERENT $p$ VALUES UNDER THE REQUIREMENT OF 99.999\% SIPV6 TRANSLATOR RELIABILITY $\left(c_{v}=1, \lambda=0.5 \mu\right)$

\begin{tabular}{c|c|c|c}
\hline $\begin{array}{c}\text { Type of } \\
\text { TrGW }(p)\end{array}$ & $\begin{array}{c}\text { Number of } \\
\text { Required } \\
\text { TrGWs }(n)\end{array}$ & $\begin{array}{c}\text { RTP Packet } \\
\text { Processing } \\
\text { Time } \overline{R_{I}}(1 / \mu)\end{array}$ & $\begin{array}{c}\text { RTP Packet } \\
\text { Processing } \\
\text { Time } \overline{R_{I I}} \\
(1 / \mu)\end{array}$ \\
\hline 0.997 & 2 & 1.337321 & 1.07250 \\
\hline 0.99 & 3 & 1.204158 & 1.008138 \\
\hline 0.95 & 4 & 1.155642 & 1.002837 \\
\hline 0.90 & 5 & 1.130206 & 1.001624 \\
\hline 0.85 & 7 & 1.095392 & 1.000252 \\
\hline 0.8 & 8 & 1.088851 & 1.000244 \\
\hline
\end{tabular}

increases. Furthermore, the increasing rates of $\overline{R_{I}}$ and $\overline{R_{I I}}$ are larger for $p=0.8$ than those for $p=0.9$. For Configuration $\mathbf{I}$, only the first incoming RTP packet of each RTP session is assigned to a TrGW by the IMS-ALG, and the subsequent RTP packets of the same RTP session are sent to the same TrGW as the first packet. Therefore, comparing Configuration I with Configuration II, the increasing rate of $\overline{R_{I}}$ is larger than that of $\overline{R_{I I}}$ (see Figs. 7, 8, and Table II). Based on the above discussion, Configuration II is better than Configuration I in terms of the queueing effect (i.e., the $\bar{R}$ measure). However, we should point out that the forwarding engine in Configuration II can be a bottleneck that we do not evaluate explicitly in our study. 
In order to meet the telecom-grade requirement, $99.999 \%$ reliability for the SIPv6 translator should be achieved. Table II illustrates the effect of $p$ on the number $n$ of the required TrGW and the expected response times $\overline{R_{I}}$ and $\overline{R_{I I}}$ to achieve $99.999 \%$ of SIPv6 translator reliability, where $\lambda=0.5 \mu$ and $c_{v}=1.0$. When $p=0.8$, a SIPv6 translator system requires eight $\operatorname{TrGWs}$ to achieve $99.999 \%$ reliability, and its expected response time is about $1 / \mu$. On the other hand, when $p=0.997$, only two TrGWs are needed, but the expected response time increases to $1.07 / \mu$.

The above analysis illustrates how to select the number and the types (in terms of reliability) of TrGWs to achieve telecomgrade performance for the SIPv6 translator.

\section{CONCLUSIONS}

Based on 3GPP 23.228 [1], we proposed an IPv4-IPv6 translation mechanism (i.e., SIPv6 translator) that supports IPv6 and IPv4 interworking infrastructures to provide an overlay network for transparent SIP application deployment. To achieve highly reliable telecom-grade requirement, the SIPv6 translator utilizes multiple TrGWs or single TrGW with multiple processors to effectively process the RTP packets. This paper described the architecture and operations of the SIPv6 translator. Then, we evaluated the effect of $n$ (i.e., the number of TrGWs/processors), $p$ (i.e., the steady-state probability that a $\mathrm{TrGW} /$ processor is operational), $\lambda$ (the net RTP packet arrival rate) and $c_{v}$ (i.e., the coefficient of variation of the RTP packet processing time $t_{s}$ ) on the expected RTP packet response times $\overline{R_{I}}$ and $\overline{R_{I I}}$.

Our study provided guidelines to configure the SIPv6 translator to achieve telecom-grade requirement. For example, our numerical examples indicate that for a fixed $p$, when $\mathrm{n} \geq 5$, adding more processors (TrGWs) only insignificantly improves the response time performance.

\section{REFERENCES}

[1] "Technical Specification Group Services and Systems Aspects; IP Multimedia Subsystem Stage 2," 3GPP. 3rd Generation Partnership Project, Tech. Spec. 3G TS 23.228, 5.1.0 (2001-06) ed., 2001.

[2] "Technical Specification Core Network; Subscriber Data Management; Stage 2," 3GPP. 3rd Generation Partnership Project, Tech. Spec. 3G TS 23.016, 4.0.0 (2001-03) ed., 2001.

[3] "Technical Specification Group Services and Systems Aspects; General Packet Radio Service (GPRS); Service Description; Stage 2," 3GPP. 3rd Generation Partnership Project, Tech. Spec. 3G TS 23.060, 4.1.0 (2001-06) ed., 2001.

[4] A.-C. Pang and Y.-K. Chen, "A study on availability of mobile database," in Proc. Int. Conf. Inf. Netw., South Korea, 2004, pp. 195-200.

[5] E. Nordmark, "Stateless IP/ICMP translation algorithm (SIIT)," IETF, RFC 2765, 2000.

[6] F. Andreasen and B. Foster, "Media gateway control protocol (MGCP)," IETF, RFC 3435, 1.0 ed., 2003.
[7] G. Tsirtsis and P. Srisuresh, "Network address translation-protocol translation (NAT-PT)," IETF, RFC 2766, 2000.

[8] H. Schulzrinne et al., "RTP: A transport protocol for real-time applications," IETF, RFC 1889, 1996.

[9] J. Rosenberg et al., "SIP: Section initiation protocol," IETF, RFC 3261, 2002.

[10] M. Handley and V. Jacobson, "SDP: Session description protocol," IETF, RFC 2327, 1998.

[11] S. M. Ross, Stochastic Processes. New York: Wiley, 1996.

[12] - Introduction to Probability Models. Orlando, FL: Harcourt, 2000.

[13] W.-E. Chen et al., "Design of SIP application level gateway for IPv6 translation," J. Internet Technol., vol. 5, no. 2, pp. 147-154, 2004.

[14] Y.-B. Lin et al., "All-IP approach for third generation mobile networks," IEEE Netw., vol. 16, no. 5, pp. 8-19, 2002.

[15] Y.-.B Lin and I. Chlamtac, Wireless and Mobile Network Architectures. New York: Wiley, 2001.

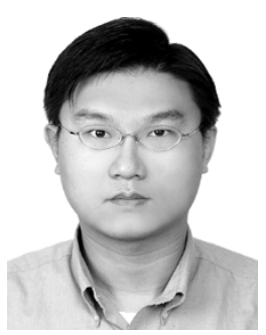

Whai-En Chen (M'03) received the B.S. degree in electric engineering from Tam Kang University, Taipei, Taiwan, R.O.C., in 1997 and the Ph.D. degree in computer science from the National Tsing-Hua University, Hsinchu, Taiwan, R.O.C., in 2002.

He began serving as a Research Assistant Professor in the National Chiao Tung University, and joined the National Telecommunications Project to deploy a SIP-based VoIP platform in 2002. His research interests include 3G IP multimedia subsystem (IMS), SIP-based VoIP services, IPv6 translation mechanisms, and high-speed lookup/classification engines.

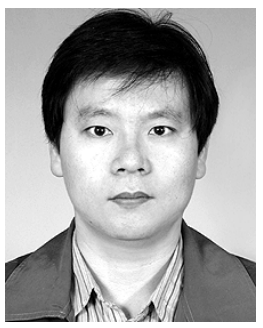

Yi-Bing Lin (M'96-SM'96-F'04) is Chair Professor and Vice President of Research and Development, National Chiao Tung University, Hsinchu, Taiwan, R.O.C. His current research interests include wireless communications and mobile computing. He is the coauthor of Wireless and Mobile Network Architecture with I. Chlamtac (New York: Wiley). He has published over 190 journal articles and more than 200 conference papers.

Dr. Lin is an Association for Computing Machinery (ACM) Fellow, an American Association for the Advancement of Science (AAAS) Fellow, and an Institute of Electrical Engineers (IEE) Fellow.

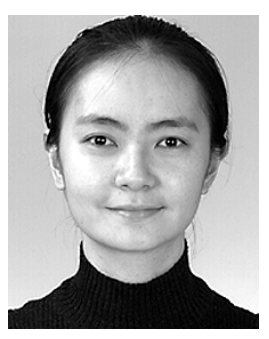

Ai-Chun Pang (M’02) was born in Hsinchu, Taiwan, R.O.C., in 1973. She received the B.S., M.S., and $\mathrm{Ph} . \mathrm{D}$. degrees in computer science and information engineering from National Chiao Tung University, Hsinchu, Taiwan, R.O.C., in 1996, 1998, and 2002, respectively.

She joined the Department of Computer Science and Information Engineering, National Taiwan University, Taipei, Taiwan, R.O.C., as an Assistant Professor in 2002. Since August 2004, she has been an Assistant Professor in the Graduate Institute of Information Networking and Multimedia, National Taiwan University. Her research interests include design and analysis of personal communications services network, mobile computing, voice-over-IP, and performance modeling. 